\title{
Analytical bound for the CCDF of Crest Factor in NC-OFDM Systems
}

\author{
Mohammed Yassine Bendimerad, Fatima Debbat, and Fethi Tarik Bendimerad
}

\begin{abstract}
Non-contiguous orthogonal frequency division multiplexing (NC-OFDM) is a promising framework for wireless communication systems due to OFDM characteristics like the spectral bandwidth efficiency, robustness to frequency selective fading channels, etc. However, it has been widely known that multicarrier signals generated by OFDM technique exhibit a large peak to average power ration (PAPR), one of the main impediment that has limited the applicability of OFDM systems. Several approaches are employed in literature to derive the PAPR distribution and thus try to reduce it in OFDM context, as Central limit theorem and modern extreme value theory. In new contexts like NC-OFDM systems when flexible access to spectrum is done with non-contiguous portions, the PAPR distribution remains generally unknown, and it is necessary to describe it, since this is one of the key design parameters. In this correspondence, we develop the PAPR bound expression in NCOFDM context.
\end{abstract}

Index terms - Complementary Cumulative Distribution Function (CCDF), Crest Factor (CF), Non-Contiguous Orthogonal Frequency Division Multiplexing (NC-OFDM), Peak to Average Power Ratio (PAPR).

\section{INTRODUCTION}

With the explosive growth of wireless multimedia applications and the demand for high data rate, dynamic spectrum access and energy efficiency become two most critical challenges for wireless communication networks, since most of the current frequency bands are already allocated and it more and more difficult to find vacant bands. In cognitive radio, context transmission systems can exploit spectrum holes by adapting system parameters according to communication environment and thus achieve reliable communication. Moreover, the flexibility of the multicarrier modulation technology can dynamically adapt spectral environments and allocate unoccupied spectrum bands, resulting in noncontiguous subcarriers being able to change their states in radio spectrum. These multicarrier technologies even if they already suffer from an intrinsic kind of interference duo to their spectral scheme often exhibit large envelope variation

Manuscript received October 13, 2015; revised December 30, 2015.

M. Y. Bendimerad and F. T. Bendimerad are with the Telecommunication Laboratory (LTT), Department of Telecommunication, University of Tlemcen, Algeria (E-mails: yassin.bendimrad@gmail.com, f.tbendimrad@gmai.com).

Fatima Debbat is with the Computer Science Department, Faculty of Technology, Mascara University, Mascara Algeria (E-mail: rst.tlcom@gmail.com) and as a result high PAPR occurs, think that can produce another kind of interference when the signal passes through analog components. In this case, the digital to analog converter and power amplifier would require a large dynamic range to avoid amplitude clipping, thus increasing both power consumption and component cost. A rigorous comparison of transmission systems in terms of energy efficiency necessitates the knowledge of statistical behavior of modulated signals, and thus the knowledge of PAPR distribution [1]. Derivation of PAPR distribution allows us to estimate a number of practical concerns such as a required linear range, the degradation of error rate and the adjacent channel interference due to clipping distortion in amplified signals [2]. In literature a number of solid theoretical analyses devoted to the distribution of the PAPR exist in OFDM context, e.g., in [3] - [6]. However in new promising contexts as NC-OFDM our knowledge about this energetic parameter is restricted. This motivates the problem of finding PAPR distribution for new waveforms, or for transmission scenarios to quantify how severe that is [11]. The distribution of PAPR bears stochastic characteristics in NC-OFDM systems as in OFDM systems and is often expressed in terms of complementary cumulative distribution function (CCDF). The CCDF can be used to estimate the bounds for the minimum number of redundancy bits required to identify the PAPR sequences and evaluate the performance of PAPR reduction schemes. In this paper, we are motivated to derive a general and accurate analytical expression of the PAPR bound in NC-OFDM systems.

The rest of the paper is outlined as follows: Section two introduce notation and non-contiguous systems. In section three the Peak to average power ratio expression for noncontiguous orthogonal frequency division multiplexing modulation (OFDM) systems is given. In section four we will review the previously reported results on the analysis of the distribution of the peak to average power ratio in OFDM systems and then go further to propose a general bound expression in this new context with it analytical estimation for practical cases. To validate the proposed expression numerical simulation results will be presented followed by conclusion in section five.

\section{NON-CONTIGUOUS SySTEM MODEL}

The basic principle of NC-OFDM system is to split a highspeed data stream into a number of slower data streams 
transmitted simultaneously over orthogonal subcarriers. These streams are generally modulated using M-ary constellation scheme. For NC-OFDM, the active subcarriers need not be contiguous and are located in unoccupied spectrum bands, the usable spectrum bands are determined by dynamic spectrum sensing and channel sounding. The inactive subcarriers located in the used spectrum band are required to be turned off to constrain the interference from the secondary users to primary users. Thus, the spectrum efficiency can be significantly improved by the NC-OFDM based cognitive radio systems. A general schematic of an NC-OFDM transmitter is shown in Figure 1. The encoder is used to modulate the binary input data to get a specific constellation generally $\mathrm{M}$-ary phase shift keying (M-PSK) or M-ary quadrature amplitude modulation (M-QAM). The cognitive radio controller senses the spectrum in order to detect used subcarriers and active bands so that these ones will be turned off during the system transmission. Subsequently, the inverse fast Fourier transform (IFFT) process converts the symbols from the frequency domain into time domain and then they are once again converted into serial format. The resulting sequence in converted to an analog signal using digital to analog converter, and then pass through radio frequency chain, which amplifies the signal and upconverts it to the desired center frequency.

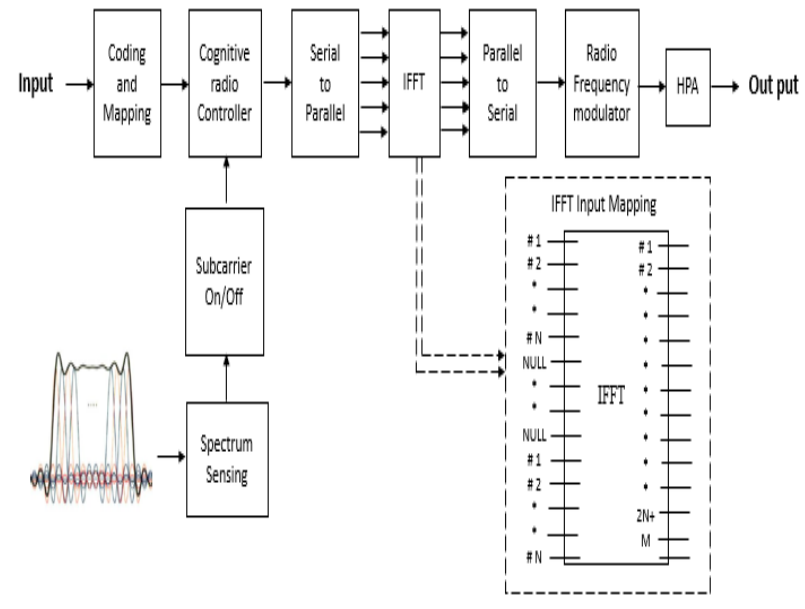

Fig .1 Typical Non Contiguous Transmitter

One of the most important elements of the NC-OFDM system is the ability to measure, sense, learn and be aware of important operating conditions. In addition, the system should be aware of user requirements and applications, available networks infrastructures and nodes, local policies and other operating restrictions [7]. In OFDM systems, conversion from time domain to frequency domain is achieved by using FFT. Hence, all the point in the time-frequency grid of the OFDM system's operating band can be scanned without any extra hardware or computation thanks to the hardware reuse of FFT cores; using this, the selection of bins that are available for exploitation can be carried out using simple hypothesis testing [7]. After the identification of active bands of licensed users (see Figure.2), a spectrum shaping process allows cognitive users to freely use the available bands in the spectrum (the unused ones in Figure.2) in a fast and efficient way.

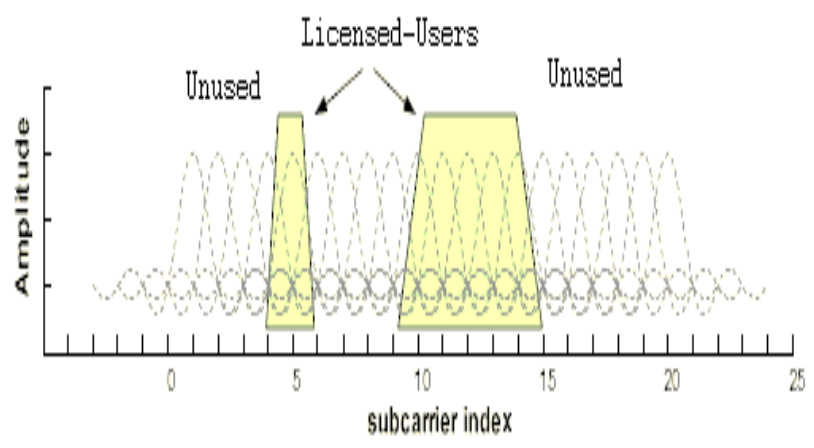

Fig 2. Spectrum sensing of unused bands

\section{PAPR DEFINITION}

The complex envelope of a baseband NC-OFDM signal, consisting off all $\mathrm{L}$ contiguous subcarriers over a time interval $[0, \mathrm{~T}]$, is given by [8]:

$$
s(t)=\frac{1}{\sqrt{L}} \sum_{k=0}^{L-1} A_{k} e^{j 2 \pi k t / T}
$$

Where $A_{k}$ is the symbol of the $k^{\text {th }}$ subcarrier, i.e. $A_{k}=1 \angle \theta_{k}$ with $\theta_{k} \in\{0, \pi\}$ for BPSK constellation and $\theta_{k} \in\left\{0, \frac{\pi}{2}, \pi_{v} \frac{3 \pi}{2}\right\}$ for QPSK constellation, $\mathrm{T}$ is the OFDM symbol duration, and $j=\sqrt{-1}$. The symbol of the $k^{\text {th }}$ inactivated subcarrier is $A_{k}=0$. For an NC-OFDM system represented by two portions of active subcarriers with the same number of subcarriers $N$ separated by a frequency lag $f_{0}$ as mentioned in Figure.3, and for a number of total subcarriers equal to the subcarriers $L$ of the equivalent OFDM system, equation (1) can be rewritten as:

$$
s(t)=\frac{1}{\sqrt{L}} \sum_{k=0}^{N-1} A_{k} e^{j 2 \pi h t / T}+\frac{1}{\sqrt{L}} \sum_{k=0}^{N-1} B_{k} e^{j 2 \pi h t / T} e^{j 2 \pi f_{6} t}
$$

Where the number active subcarriers $2 N<L$ the number of OFDM subcarriers. $f_{0}$ represents the frequency lag between the two active blocs and it vary depending on available operating conditions.

$$
\begin{gathered}
s(t)=\frac{1}{\sqrt{L}} \sum_{k=0}^{N-1}\left(A_{k} e^{j 2 \pi h t / T}+B_{k} e^{j 2 \pi h t / T} e^{j 2 \pi f_{0} t}\right) \\
s(t)=\frac{1}{\sqrt{L}} \sum_{k=0}^{N-1} A_{k} e^{\frac{j 2 \pi h t}{T}}\left(1+\frac{B_{k}}{A_{k}} e^{j 2 \pi f_{6} t}\right)
\end{gathered}
$$

The expression (4) can be generalized to a great number of active spectral blocs (blocs in gray on figure 3), given that they possess the same number of subcarriers and the total number of active subcarriers remains always less then $L$. therefore equation 4 yields: 


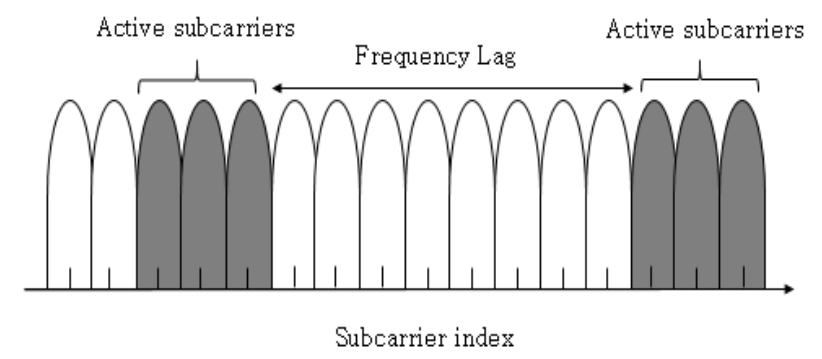

Fig. 3 NC-OFDM system's spectrum

$s(t)=\frac{1}{\sqrt{L}} \sum_{k=0}^{N-1} A_{k} e^{\frac{j 2 \pi h t}{T}}\left(1+\frac{B_{k}}{A_{k}} e^{j 2 \pi f_{6} t}+\frac{C_{k}}{A_{k}} e^{j 2 \pi f_{1} t}+\frac{D_{k}}{A_{k}} e^{j 2 \pi f_{2} t}\right)$

Where $f_{0}, f_{1}, f_{2}, \ldots$, are respectively the lags between the first active bloc and the next ones, i.e. $f_{2}$ is the frequency lag between the first bloc and the third one. The PAPR is defined as the ratio between the maximum instantaneous power and the average power:

$$
\operatorname{PAPR}(s(t))=\frac{\max _{0 \leq t \leq T}|s(t)|^{2}}{E\left\{|s(t)|^{2}\right\}}=\frac{\max _{0 \leq t \leq T}|s(t)|^{I}}{P_{\text {mean }}}
$$

The continuous time PAPR of $s(t)$ can be approximated using the discrete time PAPR. It has been shown that an oversampling factor of four is sufficient to estimate the continuous PAPR of a BPSK system. Using the Parseval's relationship the average power of the NC-OFDM signal for the case of MPSK constellation is given by (see Appendix B):

$$
\begin{aligned}
E\left[|s(t)|^{2}\right\} & =E\left\{\left|\frac{1}{\sqrt{L}} \sum_{k=0}^{L-1} A_{k} e^{\frac{j 2 \pi h t}{T}}\right|^{2}\right\} \\
& =\frac{1}{L} \sum_{k=0}^{L-1} E\left\{\left|A_{k}\right|^{2}\right\} \\
& =\frac{P}{L}
\end{aligned}
$$

Where $P$ is the number of active subcarriers, i.e. for the case of equation.4, $P$ is equal to $2 N$. The PAPR for the case of two blocs of active subcarriers separated by a frequency lag $f_{0}$ where the blocs contain the same number of subcarriers $N$ :

$$
\operatorname{PAPR}(s(t))=\frac{\max _{0 \leq t s r}\left\{\left(\left|\frac{1}{\sqrt{N}} \sum_{0}^{N-1} A_{k} e^{j 2 \pi f_{k} t}\right|^{-3} \cdot\left|\left(1+\frac{B_{k}}{A_{k}} \cdot e^{j 2 \pi f_{0} t}\right)\right|^{2}\right\}\right.}{E\left\{|s(t)|^{2}\right\}}
$$

$$
=\frac{L}{2 N^{2}} \max _{\cos t s T}\left\{\left|\sum_{0}^{N-1} A_{K^{2}} e^{j 2 \pi f_{L} t}\right|^{2} \cdot\left|\left(1+\frac{B_{k}}{A_{k}} \times e^{j 2 \pi f_{6} t}\right)\right|^{2}\right\}
$$

Furthermore, it known that the PAPR of an MPSK OFDM signal is always less than or equal to $L$, where $L$ is the total number of subcarriers [8]. in [9] NC-OFDM signal with $P$ active subcarriers is consider, where the input data are chosen from an MPSK constellation such that $\left|A_{k}\right|=1$, it is shown that the maximum value of PAPR for the MPSK modulated NCOFDM signal is equal to the number of active subcarriers $P$, regardless of the total number of subcarriers $\mathrm{L}$.

$$
\operatorname{PAPR}(s(t)) \leq P
$$

From equation (10) of PAPR in NC-OFDM context with two blocs for active subcarriers, we have:

$$
\operatorname{PAPR}(\xi(f)) \leq \frac{L}{2 N^{2}}\left\{\max _{0 S T S T}\left|\sum_{0}^{N-1} A_{k} e^{j 2 \pi f_{k} t}\right|^{2} \max _{0 S T S T}\left|\left(1+\frac{B_{k}}{A_{k}} \cdot e^{j 2 \pi f_{0} t}\right)\right|^{2}\right\}
$$

From equation (9), the mean power of the OFDM signal represented by one bloc of $N$ active subcarriers $P_{\text {maan }}=N / L$, therefore, the upper bound of the PAPR of the NC-OFDM signal depends directly on the PAPR of the equivalent OFDM signal of the portion of $N$ active subcarriers:

$\operatorname{PAPR}(s(t)) \leq \frac{1}{2 N}\left\{\right.$ PAPR $\left._{\text {OSDN }} \max _{\text {OStST }}\left|\left(1+\frac{B_{k}}{A_{K}}, e^{j 2 \pi f_{6} t}\right)\right|^{2}\right\}$

Where PAPR $_{\text {OFDM }}$ is the PAPR for the first bloc of $N$ active subcarriers, $A_{k}=1 \angle \theta_{k}$ and $B_{k}=1 \angle \varphi_{k}$. For the special case when $\angle \theta_{k}=\angle \varphi_{k}$, we have:

$$
\operatorname{PAPR}(s(t)) \leq \frac{2}{N} P A P R_{\text {ORDM }}
$$

\section{Statistical ANALYSIS OF PAPR}

The distribution behavior of the PAPR bears stochastic characteristics in OFDM systems, and is often expressed in term of complementary cumulative distribution function (CCDF). The CCDF denotes the probability that PAPR exceeds a given threshold. The same thing is expected in NCOFDM system since it can be viewed as several OFDM subblocks. The CCDF can be used to estimate the bounds of the minimum number of redundancy bits required to identify the PAPR sequences and evaluate the performance of PAPR reduction schemes. We can also directly apply distribution of PAPR to calculate bit error rate (BER) and estimate achievable information rate [16][18][19]. In this section, we present the PAPR distribution of the PAPR of the baseband discrete time NC-OFDM signal samples at the Nyquist rate. These samples may be alternatively obtained by the L-point IDFT with the L complex inputs $A_{\mathfrak{k}}$. Generally an oversampling should always be considered in the analysis of discrete time signal to better estimate the signal fluctuations. It has been shown that a sufficient accurate result can be obtained when the oversampling factor exceed 4 [10]. Let $s(t)=x(t)+j y(t)$ and $s_{l}$ denotes the complex sample. $x_{m}, y_{m}$ denote respectively the samples of the in-phase and in-quadrature components. 


\section{A. PAPR Analysis for OFDM Systems}

Assuming the processes acting on the in-phase and quadrature components of the complex envelope of the OFDM signal are independent and identically distributed (i.i.d.). As the number of subcarriers $N$ increases, the central limit theorem (CLT) can be invoked (see Appendix A). This theorem states that under certain conditions the probability of the sum of scaled mean random variables converges to the normal distribution, so that the in-phase and quadrature components tend to zero mean Gaussian variables with variance $\sigma^{2}$. The envelope, corresponding to the magnitude of the uncorrelated in-phase and in-quadrature components becomes in this case Rayleigh distributed with parameter $\sigma$ [14]. Therefore, the cumulative distribution function (CDF) of the PAPR is given by:

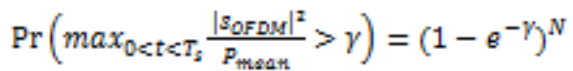

The expression obtained above is generally called the conventional distribution of the PAPR of an OFDM signal sampled at the Nyquist rate. This expression even if it yields a maximum samples of OFDM signal, it underestimates the distribution of the PAPR for the band limited OFDM signal since the samples of the signal don't necessary correspond to the maximum peaks. Afterwards, an empirical approximation of the CCDF of PAPR has been proposed, defined as:

$$
\operatorname{Pr}\left(\max _{\text {Detex }} \frac{\left|s_{\text {OFDM }}\right|^{2}}{P_{\text {mean }}}>\gamma\right)=\left(1-e^{-\gamma}\right)^{2.2 N}
$$

However equation (16) lacks theoretical validation and also yields discrepancies with the results for a large number of subcarriers. In [13] some bounds analysis has been developed in the context of OFDM systems for both dependent and independent subcarriers. An analytical CCDF expression of the PAPR has been developed in [15]; supposing that the random processes $x(t)$ and $y(t)$ are stationary in wide sense with a correlation time $T_{c}$ and the correlation may rapidly decreases as the interval of observation exceeds the correlation time, the successive peaks may have a temporal separation larger than $T_{c}$ with high probability. Therefore, the values of in-phase and inquadrature components at the sample times of interest "peaks" are statistically independent since they are uncorrelated and jointly Gaussian. This approach is called the level-crossing rate approximation of the peak distribution (see Appendix C). It mentions that by a proper selection of the threshold we may assume that the upward crossing has a single peak above the threshold level. Indeed for a for large number of subcarriers, the threshold can be chosen such that the probability that all the peaks are less than the threshold becomes negligible, therefore the conditional probability that the peak of the OFDM signals exceeds the threshold is very small. In this case, the CCDF of PAPR is given by:

$$
\operatorname{Pr}\left(\max _{\text {Detex }} \frac{\|\left. s_{\text {DFDM }}\right|^{2}}{P_{\text {msat }}}>\gamma\right)=1-\exp \left\{-N \sqrt{\frac{\pi}{a}}\right\}
$$

Another approximation of the CCDF of the PAPR for OFDM signals based on the extreme value theory is:

$$
\operatorname{Pr}\left(\max _{0<t e T_{s}} \frac{\left|s_{\text {OFDM }}\right|^{2}}{P_{\text {mWe }}}>\gamma\right)=1-\exp \left\{-N e^{-\gamma} \sqrt{\frac{\pi}{a} \log N}\right\}
$$

It is worth to note that all established analytical results so far consider that all subcarriers are active with equal power distribution. However, in normalized standards like IEEE 802.11, the OFDM waveform consist of two types of subcarriers: active subcarriers (data, channel estimation and synchronization) and inactive subcarriers (guard bands). Therefore, it is indeed needed to develop a more accurate theoretical PAPR distribution for those practical contexts [12].

\section{B. PAPR Proposed Bound}

The mean of the random variable PAPR is given by:

$$
E\{P A P R(x)\}=\int_{-\infty}^{+=0} \operatorname{PAPR}(x) \cdot \frac{\partial P r(P A P R z Y)}{\partial x} d x
$$

Where $x \equiv x_{k}$ represents the vector of complex symbols from a given constellation. Hence for its determination, the knowledge of $f(x)=\frac{\partial P r(P A P R 2 Y)}{\partial x}$ is required. However, if the PAPR is concentrated near its mean, then $\operatorname{E}\{\operatorname{PAPR}(x)\}$ can be expressed in terms of the moments $\mu_{n}$ of the PAPR. Suppose first that $f(x)$ is negligible outside an interval $\left(\eta-\varepsilon_{v} \eta+\varepsilon\right)$ and in this interval, $\operatorname{PAPR}(x) \approx \operatorname{PAPR}(\eta)$. In this case (16) yields:

$$
E\{P A P R(x)\}=\operatorname{PAPR}(\eta) \int_{\eta-\varepsilon}^{\eta+\varepsilon} f(x) d x \approx \operatorname{PAPR}(\eta)
$$

This estimation can be improved if $\operatorname{PAPR}(x)$ is approximated by a polynomial:

$$
\begin{aligned}
& E\{P A P R(x)\}=\operatorname{PAPR}(\eta)+\operatorname{PAPR}^{\prime}(\eta)(x-\eta)+\cdots+ \\
& \operatorname{PAPR}^{(n)}(\eta) \frac{(x-\eta)^{\mathrm{N}}}{n !}
\end{aligned}
$$

In particular, if PAPR $(x)$ is represented by a parabola, then:

$$
E\{P A P R(x)\} \approx \operatorname{PAPR}(\eta)+P A P R^{x(\eta)} \frac{\sigma^{2} P A P R}{2}
$$

Where $\sigma_{P A P R}^{2}$ is the variance of the PAPR which can be expressed in terms of the variance $\sigma^{2}$ of the code-word $X$ derived from the constellation. We maintain that $\sigma_{\text {PAPR }}^{2}=\mid$ PAPR $\left.^{3}(\eta)\right|^{2}$ as in [17]. Another case is when the PAPR $(x)$ is represented by a straight, then $E\{P A P R(x)\} \& \operatorname{PAPR}(\eta)$. This shows that the slope of PAPR has no effect on the mean of the PAPR; however it affects the deviation of the PAPR as we can show in the above expression of $\sigma_{P A P R}^{2}$.

So far, we have developed the exact expression of the mean followed by its accurate estimation used in practice, since the first one is no convenient to use. Since the PAPR is a random variable defined in the half positive axis (PAPR is the ratio of two positive quantities), one can writes for any $>0$ : 


$$
\begin{aligned}
E\{P A P R(x)\} & =\int_{0}^{+\infty} \operatorname{PAPR}(x) \cdot \frac{\partial \operatorname{Pr}(\operatorname{PAPR} \geq \gamma)}{\partial x} d x \\
& \geq \int_{Y}^{+\infty} \operatorname{PAPR}(x) \cdot \frac{\partial \operatorname{Pr}(\operatorname{PAPR} \geq \gamma)}{\partial x} d x \\
& \geq \gamma \int_{Y}^{+\infty} \frac{\partial \operatorname{Pr}(\operatorname{PAPR} \geq \gamma)}{\partial x} d x
\end{aligned}
$$$$
\operatorname{E}\{\operatorname{PAPR}(x)\} \geq \gamma \operatorname{Pr}(P A P R \geq \gamma)
$$

So by introducing equation (18), the exact upper bound can be expressed as:

$$
\begin{gathered}
\operatorname{Pr}(P A P R \geq \gamma) \leq \frac{E[P A P R(x)]}{\gamma} \\
\leq \frac{\operatorname{PAPR}(\eta)+\operatorname{PAPR}^{\prime}(\eta)(x-\eta)+\cdots+\operatorname{PAPR}^{(\mathrm{n})}(\eta) \frac{(x-\eta)^{x s}}{n !}}{\gamma}
\end{gathered}
$$

An approximation of the bound in (21) can be found by using the second-degree development of equation (19), then:

$$
\operatorname{Pr}(P A P R \geq \gamma) \leq \frac{P A P R(\eta)+P A P R^{Y^{r}\left(00 \theta^{x^{2} P A P R}\right.}}{2}
$$

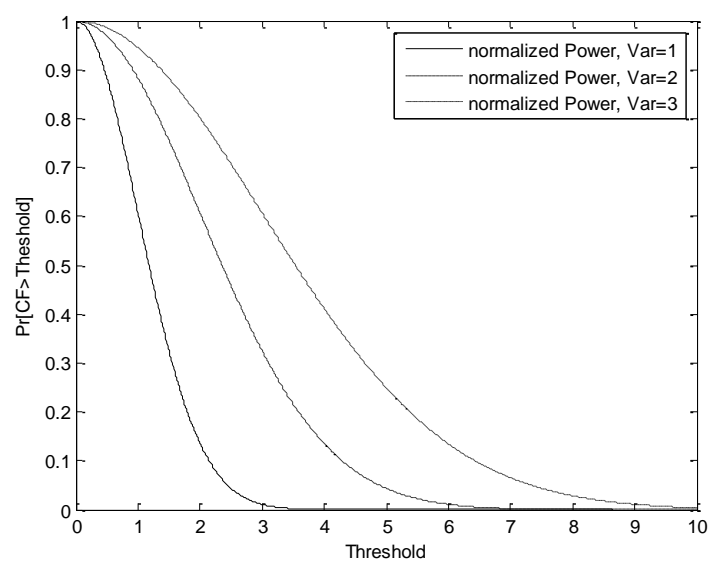

Fig. 4 Cumulative Density Function of the Crest Factor

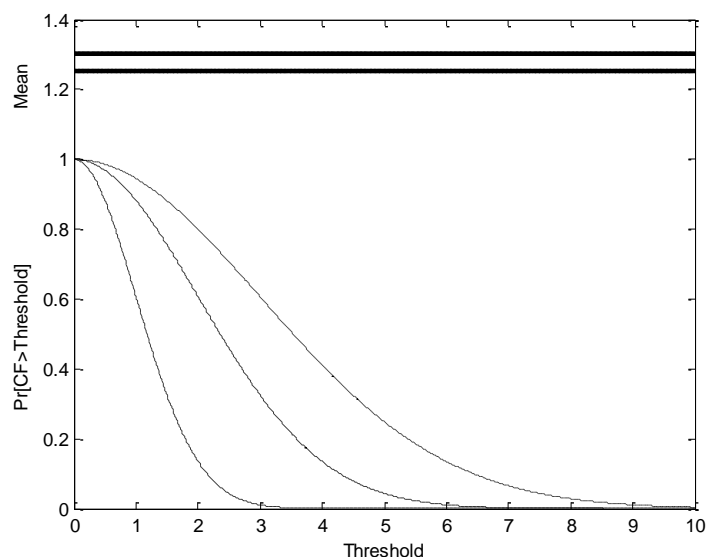

Fig. 5 CCDF of CF compared to the Normalized Mean
The complementary cumulative density functions of the crest factor are plotted in Fig.4 for different values of deviation $\sigma^{2}$. It is worth to note that the curves are simulated by taking in account only the shape of the statistical behavior of the crest factor, and not all characteristics of the $\mathrm{CF}$ in a general OFDM systems (constellation...), something that can be different in values, not in shape. We can see that more the deviation tend to increase, the concavity of the curve will be larger. However the surface under the curves remain the same.

In Fig.5 the inequalities developed in equation (20) and (21) are shown, the figure compares between the normalized mean of the crest factor and the correspondent complementary cumulative density function, it is evident from the plotted curves that for the case when the deviation equals 1 and 1.2, the normalized mean is 1.23 and 1.3 respectively see Fig.5. Therefore, the CCDF remains always under the normalized mean since this one tends to increase as the deviation augments.

\section{CONCLUDING REMARKS}

In this paper, we have proposed an analytical bound for the complementary cumulative density function (CCDF) of the Peak to Average Power ratio or the equivalent Crest Factor in NC-OFDM systems. The proposed bound is developed based on the probability theory. Computer simulations have been conducted to validate the analytical results, showing a good match between the proposed upper bound and the simulation results.

\section{APPENDIX}

\section{Berry-Essen Inequality}

In probability theory, the central limit theorem states that, under certain circumstances, the probability distribution of the sum of scaled mean random variables converges to a normal distribution as the sum increase to infinity. Under stronger assumptions, the Berry-Essen theorem, or Berry-Essen inequality, specifies the rate at which this convergence takes place by giving a bound on the maximal error of approximation between the normal distribution and the true distribution of the scaled random variables mean. Let $x_{1}, x_{2}$, $\ldots . . ., x_{n}$ be $n$ scaled mean random variables. The Berry-Essen inequality states that: There exists a positive constant $C$ such that if $x_{1}, x_{2}, \ldots \ldots, x_{n}$ are independent and identically distributed random variables with:

$$
E\left\{x_{1}\right\}=0, E\left\{x_{1}{ }^{2}\right\}=\sigma^{2}>0, \text { and } E\left\{\left|x_{1}\right|^{2}\right\}=\rho<\infty
$$

And if we define the sample mean $Y_{\mathrm{n}}$ by:

$$
Y_{n}=\frac{x_{1}, x_{2 n \ldots x w} x_{n}}{n}
$$

With $F_{\mathrm{n}}$ the cumulative distribution function of $\frac{\mathbb{Y}_{\mathrm{in}}}{\sigma^{2} \sqrt{n}}$ and $\varphi$ the cumulative distribution function of the standard normal distribution, then: 


$$
\left|F_{n}(x)-\varphi(x)\right| \leq \frac{C_{x} \rho}{\sigma^{3} \sqrt{n}}
$$

\section{Average Power Expression}

The average power for the OFDM symbol is calculated as follow:

$$
\begin{aligned}
& P_{\text {mean }}=\frac{1}{2 t_{0}} \lim _{t_{0 \rightarrow+\infty}} \int_{-t_{0}}^{+t_{0}} E\left(|s(t)|^{2}\right) d t
\end{aligned}
$$

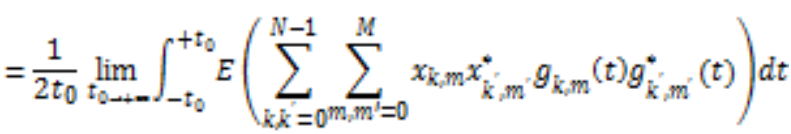

Where $g(t)=\frac{1}{\sqrt{N}} e^{\frac{\min \left(t-\frac{d}{2}\right)}{T R}} * \Pi(t)$ (see equation (4)).

Since $x_{k m}$ are uncorrelated and central limit theorem can be invoked for the OFDM base band signals as $\mathrm{N}$ increase, one may writes:

$$
\begin{aligned}
P_{\text {mean }} & =\frac{1}{2 t_{0}} \lim _{t_{0 \rightarrow+\infty}} \int_{-t_{0}}^{+t_{0}} \sum_{k=0}^{N-1} \sum_{m=0}^{M} \sigma^{2}\left|g_{k m}(t)\right|^{2} d t \\
& =\sigma^{2} \frac{1}{2 t_{0} t_{0 \rightarrow+\infty}} \sum_{k=0}^{N-1} \int_{-t_{0}}^{+t_{0}} \sum_{m}^{M}\left|g_{k m}(t)\right|^{2} d t
\end{aligned}
$$

Let us put $t_{0}=\frac{K T}{2}{ }_{0} K \in N$

$$
\begin{aligned}
P_{\text {mean }}= & \frac{\sigma^{2}}{T} \lim _{K \rightarrow+\infty=0} \frac{1}{K} \sum_{k=0}^{N-1} \int_{\frac{K T}{2}}^{+\frac{K T}{2}} \sum_{m=0}^{M}\left|g_{k m}(t)\right|^{2} d t \\
& =\frac{\sigma^{2}}{T} \sum_{k=0}^{N-1} \int_{\frac{T}{2}}^{+\frac{T}{2}} \sum_{m=0}^{M-1}\left|g_{k}(t-m T)\right|^{2} d t
\end{aligned}
$$

Since the OFDM symbol is of period $\mathrm{T}$ and the function $g_{\mathrm{km}}(t)$ is periodic with the same period.

$$
P_{\text {mean }}=\frac{\sigma^{2}}{T} \sum_{k=0}^{N-1} \sum_{m=0}^{M-1} \int_{m-\frac{T}{2}}^{m+\frac{T}{2}}\left|g_{k}(t-m T)\right|^{2} d t
$$

$$
\begin{gathered}
=\frac{\sigma^{2}}{T} \sum_{k=0}^{N-1} \int_{-\infty}^{+\infty}\left|g_{k}(t)\right|^{2} d t \\
=\frac{\sigma^{2}}{T} \sum_{k=0}^{N-1}\left\|g_{k}(t)\right\|^{2} d t
\end{gathered}
$$

\section{Level Crossing Rate of OFDM Signal}

The envelop of the OFDM signal is given by:

$$
s^{s}(t)=\sqrt{\frac{x^{2}(t)+y^{2}(t)}{2 \cdot \sigma^{2}}}
$$

The processes $x(t)$ and $y(t)$ are independent and stationary Gaussian with zero mean and variance $\sigma^{2}$. We can assume that the processes $x(t)$ and $y(t)$ are also Gaussian random processes with zero mean and variance $\sigma_{\tilde{w}}^{2}=E\left[x(t)^{2}\right]$. The joint probability density function for the processes $x_{v} y_{0} \tilde{x}_{v} \dot{y}$ is written as :

$$
f_{x y}(T)=\frac{1}{\sqrt{2 \pi|R|}} e^{-\frac{1}{2} T \cdot R^{-1} T^{-1}}
$$

This PFD has the shape of a Gaussian probability density function with $\mathrm{T}$ and $\mathrm{R}$ two matrix that represent process vector $\left[x_{v} \tilde{x}_{v} y_{*} \tilde{y}\right]$ and the covariance matrix, respectively.

$$
R=\left[\begin{array}{cccc}
\sigma^{2} & 0 & 0 & 0 \\
0 & \sigma^{2} \tilde{X} & 0 & 0 \\
0 & 0 & \sigma^{2} & 0 \\
0 & 0 & 0 & \sigma_{\tilde{W}}^{2}
\end{array}\right]
$$

In the polar coordinate the joint PDF becomes:

$$
\begin{aligned}
& f_{x y}\left(r, \hat{\tilde{x}}_{v}, \theta_{v} \dot{\theta}\right)=\frac{r^{2}}{\pi^{2} K} e^{-r^{2}-\frac{1}{K^{2}}\left(-\tilde{r}^{2}+r^{2} \tilde{\theta}^{2}\right)} \\
& K=\frac{\sigma_{\tilde{z}}^{2}}{\sigma^{2}}
\end{aligned}
$$

Where the polar coordinate are defined by:

$$
\begin{aligned}
& x=\sqrt{2 \sigma^{2}{ }_{x}} r \cos (\theta) \\
& y=\sqrt{2 \sigma^{2}}{ }_{x} r \sin (\theta)
\end{aligned}
$$

Integrating the above joint PDF expressed in the polar coordinate by $\theta$ from 0 to $2 \pi$ and $\dot{\theta}$ from $-\infty$ to $+\infty$, we obtained the joint PDF of $r$ and $\tilde{r}$ :

$$
f_{r}\left(r_{x} \dot{r}\right)=2 r e^{-r^{2}} \frac{1}{\sqrt{\pi K}} e^{\frac{-\dot{\gamma}^{2}}{K}}
$$

The level crossing rate, which is the mean number of positive crossings of the process representing the envelop of the OFDM signal at the level rate $r=y$ is given by: 


$$
\begin{gathered}
\theta^{+}(\gamma)=\int_{0}^{\infty} \tilde{r} f_{r}(\gamma, \dot{r}) d \dot{r} \\
\vartheta^{+}(\gamma)=\int_{0}^{\infty} \dot{\gamma} 2 \gamma e^{-\gamma^{2}} \frac{1}{\sqrt{\pi K}} e^{\frac{-\tilde{r}^{2}}{K}} d r=\sqrt{\frac{K}{\pi}} \gamma_{*} e^{-\gamma^{2}}
\end{gathered}
$$

\section{ACKNOWLEDGMENT}

The authors are indebted to the reviewers for their reading of the manuscript and their worthy comments.

\section{REFERENCES}

[1] M.Tanahashi and H.Ochiai,"On the distribution of instantaneous power in single-Carrier signals," IEEE Trans. Commun, vol.9, no.3, pp 12071215, Mar.2010.

[2] H. Ochiai, "Power efficiency comparison of OFDM and single-carrier signals," in Proc. IEEE VTC'02 Fall, pp. 899-903, Sept. 2002.

[3] P.Assimakopoulos, A.Nkansah, N.J.Gomes, and D.Wake" Statistical distribution of EVM measurements for direct-modulation radio-overfiber links transportating OFDM signals," IEEE Trans. Microwave theory and techniques, DOI: 10.1109/TMTT.2013.2247615, Jan.2013.

[4] N. Dinur and D. Wulich, "Peak-to-average power ratio in high-order OFDM," IEEE Trans. Commun., vol. 49, pp. 1063-1072, June 2001.

[5] H. G. Myung, J. Lim, and D. J. Goodman, "Peak-to-average power ratio of single carrier FDMA signals with pulse shaping," in Proc. IEEE PIMRC'06, Sept. 2006

[6] D.Guel," Étude de nouvelles techniques de réduction du facteur de crête à compatibilité descendante pour systèmes multiporteuses," $\mathrm{PhD}$ dissertation, University fo Rennes 1, Nov.2009.

[7] A. D. S. Jayalath and C. Tellambura, "Adaptive PTS approach for reduction of peakto-average power ratio of OFDM signal," Electronics Letters, vol. 36, pp. 1226-1228, July 2000.

[8] R. van Nee and A. de Wild, "Reducing the peak-to-average power ratio of OFDM," in Proc. 48th IEEE Vehicular Technology Conference VTC 98, vol. 3, pp. 2072-2076, 18-21 May 1998.

[9] S.H.Han, J.M.Cioffi, and J.H. Lee, "Tone injection with hexagonal constellation for peak-to-average power ratio reduction in OFDM," IEEE Communication Letters, vol. 10, pp. 646-648, September 2006.

[10] T.Jiang and Y.Wu," An overview: Peak-to-average power ratio reduction techniques for OFDM signals," IEEE Trans. on broadcasting, vol.54, $\mathrm{N}^{\circ} .2$, June 2008.

[11] M.Sharif and B.Hassibi," On multicarrier signals where the PMEPR of a random codeword is asymptotically log n" IEEE Trans. information theory, vol.50, no.5, pp.895-903, May.2004.

[12] G.Santella and F.Mazzenga, "A hybrid analytical simulation procedure for performance in M-QAM-OFDM schemes in presence of nonlinear distortions," IEEE Trans. Veh. Technol., vol. 47, pp. 142 151, Feb.1998.

[13] G.Wunder and H.Boch, "Upper bounds on the statistical distribution of the crest factor in OFDM transmission," IEEE Trans of information theory, vol.49, pp.488-494, Feb.2003.

[14] B.Hamad and A.Matin," PAPR distribution analysis of OFDM signals with partial transmit sequence," Journal of communication, Vol.7, np.11, pp. 784-789, Nov.2012.

[15] H.Ochiai and H.Imai," On the distribution of the peak-to-average power ratio in OFDM systems," IEEE Trans.Communications, vol.49, no. 2, pp.282-289, Feb.2001.
[16] A.Powell, "On the fatigue failure of structures due to vibrations excited by random pressure fields" J.Acoust.Soc.Amer, vol. 35, no. 11, pp. 1693-1699, Nov.1963.

[17] A. Papoulis, Probability, Random Variables, and Stochastic Processes, 3rd ed. New York, NY, USA: McGraw-Hill, 1991.

[18] Tao Jiang and al." Derivation of PAPR distribution for OFDM Wireless systems based on Extreme value theory," IEEE Trans. On wireless communications, Vol.7, $\mathrm{N}^{\circ} .4$, April 2008.

[19] M.Frieze, "On the achievable information rate with peak-power limited OFDM," IEEE trans. Inform. Theory, Vol.46, $\mathrm{N}^{\circ} .11$, pp.2579-2587, Nov.2000.

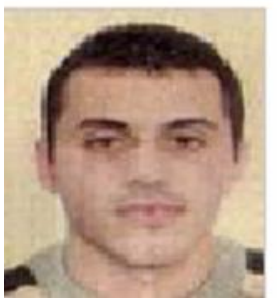

Bendimerad Mohammed Yassine and received his bachelor degree in electronics in 2010 from Department of Electrical and Electronic Engineering at the University of Tlemcen, Algeria and the Mater degree in Telecommunication Systems and Networks from the same university in 2012. He is currently researcher in Telecommunication field. His current research interests include the areas of wireless communications and corresponding signal processing.

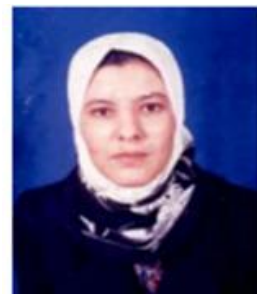

Debbat Fatima received gis bachlor degree in 1996 from Science and technology University of Oran USTO, and a master Degree in Space Technologies, in 2002 from the Space Techniques Center (CTS) Algeria and Ph.D. Degree in electronics, in 2007 from University of Tlemcen, Algeria. She is Currently Assistant at the department of computer Science in Mascara University, Algeria. Her current research interest covers Artificial Intelligence Applications, Optimization and Wireless Networks.

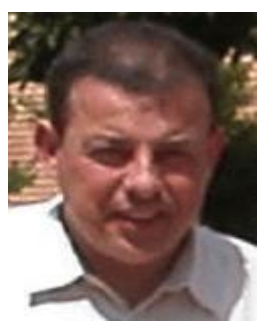

Bendimerad Fethi Tarik received his Engineering Degree in 1983 from the Science and Technology University in Oran, Algeria and his Doctorate Degree from Nice Sophia Antipolis University, France, in 1989. He is a professor and the Director of the Telecommunications Laboratory at university of Tlemcen. At the same time, he is responsible of the digital communication section. His main area of research is the microwave techniques and radiation, wireless systems and multiple antenna systems. 